\title{
EVIDENCIAS DE VALIDEZ DE UNA MEDIDA DE COMPETENCIAS GENÉRICAS
}

\author{
(EVIDENCE OF VALIDITY OF A GENERIC COMPETENCIES MEASURE)
}

María Mónica Álvarez Benítez Universidad Popular Autónoma del estado de Puebla (UPAEP), México Isabel Inmaculada Asensio-Muñoz Universidad Complutense de Madrid DOI: 10.5944/educXX1.25896

Cómo referenciar este artículo/How to reference this article:

Álvarez Benítez, M.M. \& Asensio-Muñoz, I.I. (2020). Evidencias de validez de una medida de competencias genéricas. Educación XX1, 23(2), 337-366 doi: 10.5944/educXX1.25896

Álvarez Benítez, M.M. \& Asensio-Muñoz, I.I (2020). Evidence of validity of a generic competencies measure. Educación XX1, 23(2), 337-366, doi: 10.5944/educXX1.25896

\section{RESUMEN}

El nivel de empleabilidad que consiguen las Universidades está considerado como un indicador de calidad y se relaciona estrechamente con la formación en competencias profesionales que reciben los estudiantes. Pero el desempeño profesional de los egresados depende, no solo del dominio de competencias específicas que se trabajan de manera particular e intencional en cada carrera y asignatura, sino también del desarrollo de competencias genéricas, que no siempre se consigue con los métodos y estructuras de enseñanza-aprendizaje formales. Este trabajo se centra en el estudio de las competencias genéricas más relacionadas con la autoeficacia profesional y se ubica dentro de una investigación más amplia que relaciona su desarrollo con la práctica deportiva. El propósito particular del presente artículo es aportar evidencias de validez de una medida de competencias genéricas aplicable en la Universidad, mediante el uso de 
una técnica poco convencional, como es la segmentación jerárquica. La muestra utilizada está formada por 1267 alumnos universitarios, españoles y mexicanos, deportistas y no deportistas. Con el programa SPSS se obtiene el estudio de fiabilidad y los árboles CART (Classification And Regression Trees), así como la importancia normalizada de los ítems en cuanto a su relación con el criterio de validez, que es la autopercepción competencial para el trabajo que manifiestan los encuestados. Los resultados permiten identificar y jerarquizar las competencias genéricas que, atendiendo a la percepción de los encuestados, convendría trabajar en la Universidad si se pretende mejorar sus índices de empleabilidad. Se discute la aportación metodológica novedosa que supone el empleo de la segmentación empleada como técnica empírica de obtención de evidencias de validez en el avance de la investigación en este campo.

\section{PALABRAS CLAVE}

Competencias genéricas; deporte universitario; técnicas de segmentación; validez.

\section{ABSTRACT}

The level of employability achieved by universities is considered an indicator of quality and is closely related to the professional competencies received by students. The professional performance of graduates depends on the mastery of specific competencies, developed in particular and intentional ways in each degree and subject, but also on the development of generic competencies that are not always achieved with the methods and structures of formal teaching-learning. This study focuses on the study of these generic competences most often related to professional self-efficacy and is located within a wider research that links its development to the practice of sports. The specific objective of this article is to provide evidence of validity of a generic competencies measure applicable in universities using an unconventional technique, such as hierarchical segmentation. The sample consists of 1,267 university students, both Spanish and Mexican, athletes and non-athletes. The CART algorithm (Classification and Regression Trees) is obtained using the SPSS program and the importance of standardized items in terms of their relationship with the validation criterion, which is the self-perception of competency for the job manifested by the respondents. The results allow definition of the generic competencies that should be developed at the university in order to improve its employability rates. The innovative methodological contribution that involves segmentation 
methodology employed to obtain evidence of validity in the advancement of research in this field, is discussed.

\section{KEYWORDS} validity.

Generic competencies; college sports; segmentation techniques;

\section{INT RODUCCIÓN}

\section{Competencias genéricas}

La formación en competencias en la Universidad está estrechamente vinculada a la empleabilidad de sus egresados, que tienen que demostrar que al finalizar sus estudios han desarrollado una serie de habilidades, actitudes, valores y destrezas que les permitirán ser competentes profesionalmente. Las competencias específicas suelen conseguirse a través del trabajo en el aula o en laboratorios o mediante prácticas externas, gracias a la realización de las tareas académicas más convenientes en cada caso. Pero existen una serie de competencias genéricas o trasversales (González y González, 2008; López, León y Pérez, 2018) y más difíciles de medir y de evaluar (Castro, 2011), que no suelen trabajarse de manera sistemática en la Universidad y que sin embargo parecen esenciales en el desempeño profesional. Entre dichas competencias, las emocionales alcanzan un papel primordial, junto con otras más directamente laborales o de gestión del trabajo. La Universidad debe investigar modos de trabajar estas competencias para mejorar la empleabilidad de sus egresados. Desde nuestro planteamiento, un uso adecuado del deporte universitario podría contribuir al logro de estos objetivos de enseñanza-aprendizaje (Álvarez-Benítez, 2016).

\section{Deporte universitario}

Lo más habitual es que el deporte universitario sea considerado como una actividad de ocio y recreación, que sirve para estimular una vida sana y un clima institucional de fraternidad e incluso de identidad cultural de la institución (Borda y Ormeño, 2010). Pero hay investigaciones que van más allá en lo relativo al papel educativo del deporte y lo abordan como una actividad que promueve valores sociales y personales, dejando una huella en los practicantes que dura toda la vida (Ruiz y Cabrera, 2004; Holt \& Dunn, 2004; Artinger, et al., 2006). En esta línea, se plantea la necesidad de que las universidades españolas y latinoamericanas fomenten el deporte 
en sus instituciones (Álvarez-Benítez, 2016), como ya hacen por otra parte las universidades anglosajonas, e incorporen la potencialidad formativa del deporte para el desarrollo de competencias genéricas que, por otra parte, pueden resultar muy útiles para una mejor y mayor empleabilidad de los egresados universitarios. La investigación sobre este tema en nuestro contexto cultural es escasa y requiere de evidencias empíricas que avalen dicha relación y la validez de las medidas en que se apoya.

\section{Validez}

Entre los requisitos científicos de una investigación social en donde se utiliza un instrumento de medida, ocupa un papel principal el proceso de verificación de la validez de las medidas obtenidas. Este proceso de validación consiste básicamente en reunir evidencias para comprobar que el instrumento empleado para medir, cuestionario o test, cumple con el propósito para el que fue diseñado (Sireci \& Padilla, 2014). En el enfoque más tradicional se emplea el grado de acuerdo entre expertos como aproximación a la validez de contenido, el análisis de regresión, como técnica de obtención de evidencias de validez de criterio, y técnicas multivariadas, como el análisis factorial exploratorio (AFE) o el análisis factorial confirmatorio (AFC), como medios de aproximación a la validación de constructo centrada en la estructura interna de aquello que se mide.

En la revisión más actual de los Standards de la American Educational Research Association (AERA), la American Psychological Association (APA) y el National Council on Measurement in Education (NCME) (2014) se insiste en que la validez es un concepto unitario, por lo que las diversas fuentes de evidencia ilustran diferentes aspectos de la validez, pero no representan distintos tipos de la misma. La validez se concibe así como el grado en el que la evidencia acumulada apoya la interpretación de las puntuaciones que se obtienen con un instrumento de medida (test, escala, cuestionario...), teniendo en cuenta el propósito que este persigue, o lo que es lo mismo, el fin para el cual ha sido diseñado. Bajo estos presupuestos, los objetivos de la medida son los que marcan el tipo de aproximación a la validez más conveniente en cada caso

A diferencia de lo que ocurre cuando se trabaja con tests, cuyos datos permiten por lo general un uso más justificado de técnicas estadísticas altamente exigentes en cuanto a sus requisitos, cuando se trata de datos procedentes de cuestionarios o escalas, no hay pruebas de validez sensu estricto (Morales, Urosa, y Blanco, 2003), sino diferentes aproximaciones metodológicas y, en ese abanico de diversidad, el uso de la segmentación jerárquica supone una aportación novedosa, por lo infrecuente de su empleo 
a pesar de su versatilidad que permite una mayor adecuación al tipo de variables que más habitualmente entran a formar parte de la investigación educativa.

\section{Técnicas de segmentación}

El término segmentación se refiere al algoritmo o proceso estadístico, secuencial descendente, que lleva a la identificación de segmentos o subgrupos homogéneos en una variable dependiente, atendiendo a las características de los sujetos estudiados en una selección de las variables independientes y de acuerdo con unas reglas. Su uso se está extendiendo y se va reclamando como más necesario a medida que, gracias al desarrollo tecnológico, se ha incrementado de manera exponencial el número de datos de los que se dispone de los usuarios de los distintos productos y servicios de consumo (big data), así como la capacidad de almacenamiento de los mismos (data warehouses). La explotación de estos datos, su transformación en información, mediante procesos de minería de datos (data mining) o knowledge discovery in databases $(K D D)$ y la gestión de este conocimiento con fines comerciales forman parte fundamental del desarrollo de las estrategias que configuran lo que se denomina en marketing "inteligencia de negocio" (Business Intelligence). La minería de datos en educación más conocida por sus siglas en inglés EDM (Educational Data Mining) (Romero y Ventura, 2010) es una corriente internacional en la que se inscriben los trabajos que aplican las técnicas de minería de datos en el ámbito educativo. En general, la minería de datos es un proceso complejo que comprende un conjunto de técnicas avanzadas, en las que confluyen las procedentes del campo de la inteligencia artificial y las aportadas desde la estadística, para el descubrimiento de relaciones significativas y de nuevos patrones, perfiles y tendencias al examinar grandes cantidades de datos. Entre las técnicas estadísticas avanzadas que se emplean habitualmente en minería de datos para la obtención de información pertinente y relevante están las de reducción de la dimensionalidad, las de modelización y las de clasificación y de predicción, donde se incluyen las técnicas de análisis de segmentación en las que nos centramos aquí. En este contexto, los árboles de decisión resultan muy útiles como recurso para separar, utilizando el símil procedente de la actividad minero-metalúrgica que ha servido para dotar de nombre al proceso, "la mena de la ganga" (Castro y Lizasoaín, 2012) o "el oro de la arena” (Asensio, Carpintero, López, y Expósito, 2018).

En investigación educativa, las técnicas de segmentación se presentan como una alternativa de análisis muy interesante, ya que se ajustan mejor que las tradicionalmente usadas a las características de los datos con los que solemos trabajar (Blanco, Asensio, Carpintero, Ruiz, y Expósito, 2017) 
y están demostrando ser muy útiles en evaluación de programas (Lizasoain y Joaristi, 2000; Lizasoain, Joaristi, Santiago, Lukas, Moyano, Sedano, y Munárriz, 2003), para la selección de predictores relevantes del desempeño (Martínez Abad y Chaparro, 2017; Asensio, et al., 2018), para el estudio del impacto de variables contextuales en el rendimiento educativo (Tourón, López-González, Lizasoain, Navarro, y García, 2018) o para la validación de medidas (Blanco, et al., 2017). Este último es el uso que se le confiere aquí.

\section{MÉTODO}

\section{Objetivos}

Con árboles de clasificación y regresión como aproximación metodológica a la validez, se busca reunir evidencias que avalen que el instrumento que se utiliza en esta investigación, el COMPETEST (Álvarez-Benítez, Asensio-Muñoz, y García-Ramos, 2013), aporta medidas discriminativas y útiles para la explicación de un criterio. Se busca específicamente jerarquizar la importancia de las competencias genéricas, medidas según su importancia relativa, para la discriminación entre los encuestados que se perciben a sí mismos como más y menos capacitados globalmente para el ejercicio profesional.

\section{Población y muestra}

El estudio empírico se basa en una muestra no probabilística, conformada tanto por jóvenes españoles como por mexicanos, que practicaban deporte a diferente nivel.

Para el caso de la muestra española se encuentran representados estudiantes de la Universidad Complutense de Madrid (UCM), que son deportistas de élite, practican el deporte de manera regular y no lo practican, de modo que fueron invitados a participar los deportistas que competían en todas las disciplinas deportivas del torneo interno de la UCM, así como deportistas de élite en las disciplinas deportivas en las que representan a la Universidad, además de estudiantes que se encontraban cursando el último año de estudios de su carrera, pero que no practicaban deporte o lo hacían simplemente como ejercicio lúdico o de mantenimiento físico, sin llegar a competir.

En cuanto a la muestra mexicana, esta fue obtenida principalmente en el evento nacional universitario que se lleva a cabo cada año, y que, para la fecha en que se realizó la recogida de la muestra, se celebró en la ciudad de 
Toluca, Estado de México. El objetivo principal de este evento es 1) mostrar el desarrollo competitivo de los atletas estudiantes universitarios que desempeñan la doble función de estudiar y cumplir con alguna disciplina deportiva, a la vez que 2) fomentar la práctica del deporte en los jóvenes cumpliendo con una educación integral.

Componen la muestra total utilizada en este estudio 1267 universitarios, de los que 606 son españoles y 661 mexicanos, con una edad promedio de 22.56 ( \pm 4.68 años). El 57.7\% eran mujeres y el $42.3 \%$ varones. El $69.4 \%$ de los participantes son únicamente estudiantes (no trabajan). Del total de la muestra el $92.7 \%$ eran estudiantes universitarios de los últimos cursos de grado y el $2.3 \%$ estudiantes de postgrado. En lo que se refiere a las variables relacionadas con el deporte, cabe destacar que, del total de la muestra, el $12.4 \%$ resultaron ser sedentarios y el $87.6 \%$ deportistas de diferente nivel.

\section{Instrumento}

El instrumento de medida que se ha utilizado es el cuestionario COMPETEST con escala de respuesta de 6 puntos que fue diseñado para la autovaloración de las competencias genéricas identificadas como fundamentales en el ejercicio del trabajo diario en diferentes profesiones a partir del estudio de las declaraciones expresadas por empresarios tanto mexicanos como españoles (Álvarez-Benítez, Asensio-Muñoz, y GarcíaRamos, 2013). La herramienta se elaboró con el propósito de medir las competencias que se deberían adquirir tras una formación integral, surgida de la experiencia universitaria, que comprende actividades académicas y otras como la participación en actividades deportivas. La prueba completa consta de 61 preguntas con tres dimensiones (Tabla 1), de 20 ítems cada una, además de un ítem criterio que mide la autovaloración global de la competencia para el trabajo (Álvarez-Benítez, Asensio-Muñoz, y GarcíaRamos, 2013).

Tabla 1

Estructura del cuestionario

\begin{tabular}{lll}
\hline Dimensión & \multicolumn{1}{c}{ Definición constitutiva } & \multicolumn{1}{c}{ Sub-dimensiones } \\
\hline Competencias & Capacidad para reconocer & Autoconocimiento \\
Emocionales & y controlar los propios & Motivación \\
& estados de ánimo de manera & Autocontrol \\
& consciente (IE) & Empatía \\
& & Relaciones interpersonales
\end{tabular}




\begin{tabular}{|c|c|c|}
\hline Dimensión & Definición constitutiva & Sub-dimensiones \\
\hline $\begin{array}{l}\text { Competencias } \\
\text { Directivas }\end{array}$ & $\begin{array}{l}\text { Habilidades y actitudes } \\
\text { requeridas para desempeñar } \\
\text { un puesto directivo o medio } \\
\text { alto, dependiendo de la } \\
\text { estructura y políticas de la } \\
\text { empresa. }\end{array}$ & $\begin{array}{l}\text { Pensamiento estratégico } \\
\text { Liderazgo } \\
\text { Trabajo en Equipo } \\
\text { Orientación a Resultados } \\
\text { Comunicación }\end{array}$ \\
\hline $\begin{array}{l}\text { Competencias } \\
\text { Laborales }\end{array}$ & $\begin{array}{l}\text { Habilidades y actitudes que } \\
\text { ha de dominar de manera } \\
\text { general cualquier trabajador. } \\
\text { Son las más básicas } \\
\text { requeridas para estar } \\
\text { inmerso en la dinámica } \\
\text { laboral y se manifiestan en } \\
\text { todos los puestos de trabajo, } \\
\text { desde un puesto base hasta } \\
\text { un puesto gerencial. }\end{array}$ & $\begin{array}{l}\text { Disciplina } \\
\text { Ética } \\
\text { Espíritu Emprendedor } \\
\text { Aprendizaje y Mejora Continua } \\
\text { Empeño }\end{array}$ \\
\hline
\end{tabular}

Álvarez-Benítez, Asensio-Muñoz, y García-Ramos (2013) presentan resultados psicométricos preliminares obtenidos con un grupo piloto de 288 sujetos. En dicho grupo se hizo el análisis de los ítems y se obtuvo un coeficiente de fiabilidad para la prueba completa, $\alpha=0,958$.

\section{Procedimiento de recogida y análisis de datos}

Para la aplicación del cuestionario, en el caso de la muestra española se acudió principalmente a las instalaciones deportivas, donde se entrenaba o competía en las diferentes disciplinas. La obtención de datos procedentes de la muestra mexicana se realizó especialmente en los hoteles sedes del evento nacional universitario antes referido.

Para el análisis de datos se han utilizado técnicas arborescentes o de segmentación jerárquica, entre las que cabe destacar los métodos CHAID, CART, QUEST, que se pueden realizar con SPSS y que se encuentran dentro de las técnicas de clasificación (Berlanga, Rubio, y Villá, 2013; Hair, Anderson, Tatham, \& Black, 2007). En este trabajo se emplea CART (Breiman, Friedman, Olshen, \& Stone, 1984) que consiste en un algoritmo de árbol binario muy versátil porque se ajusta a todo tipo de variables. Hay que advertir, no obstante, que es una técnica que, como todas las que se emplean en minería de datos, requiere un tamaño de muestra amplio. Berlanga, et al. (2013) aconsejan que se eviten muestras con menos de 1000 casos. 
Para la acumulación de evidencias acerca de si el COMPETEST mide competencias genéricas relacionadas con la autovaloración global de competencia para el trabajo se ha utilizado una aproximación basada en los árboles de decisión, con el objetivo de identificar qué ítems o variables del instrumento discriminan mejor entre los sujetos que se consideran globalmente competentes y los que no. Por lo tanto, la variable dependiente para este estudio es el ítem 61 (incluido en el COMPETEST como referencia o criterio) y las variables independientes los otros 60 ítems, que se meterán en los análisis en un primer momento por dimensiones y finalmente todas juntas.

\section{RESULTADOS}

Las competencias emocionales, que constituyen la primera dimensión con un $\alpha=0,881$, se describen en la tabla 2

Tabla 2

Competencias emocionales

\begin{tabular}{|c|c|c|}
\hline Ítem & Sub-dimensión & Descripción del ítem \\
\hline 1 & Autoconocimiento 1 & $\begin{array}{l}\text { Frecuencia en la toma de decisiones con } \\
\text { seguridad }\end{array}$ \\
\hline 2 & Autoconocimiento 2 & $\begin{array}{l}\text { Actuación con autonomía cuando hay } \\
\text { imprevistos }\end{array}$ \\
\hline 3 & Autoconocimiento 3 & $\begin{array}{l}\text { Reconocimiento de los propios estados de } \\
\text { ánimo }\end{array}$ \\
\hline 4 & Autoconocimiento 4 & $\begin{array}{l}\text { Identificación de los propios estados } \\
\text { emocionales no convenientes }\end{array}$ \\
\hline 5 & Autocontrol 1 & $\begin{array}{l}\text { Adecuación de las decisiones tomadas en } \\
\text { situaciones adversas }\end{array}$ \\
\hline 6 & Autocontrol 2 & $\begin{array}{l}\text { Comportamiento estable ante situaciones de } \\
\text { caos }\end{array}$ \\
\hline 7 & Autocontrol 3 & $\begin{array}{l}\text { Resistencia al desgaste físico y emocional en } \\
\text { situaciones de estrés }\end{array}$ \\
\hline 8 & Autocontrol 4 & $\begin{array}{l}\text { Contribución a la resolución de situaciones de } \\
\text { tensión en el trabajo diario }\end{array}$ \\
\hline 9 & Motivación 1 & Orientación clara a objetivos \\
\hline 10 & Motivación 2 & $\begin{array}{l}\text { Optimismo hacia el logro de metas incluso con } \\
\text { malas experiencias previas }\end{array}$ \\
\hline 11 & Motivación 3 & $\begin{array}{l}\text { Motivación de logro de metas más allá de las } \\
\text { propuestas inicialmente }\end{array}$ \\
\hline
\end{tabular}




\begin{tabular}{|c|c|c|}
\hline Ítem & Sub-dimensión & Descripción del ítem \\
\hline 12 & Motivación 4 & Interés en el trabajo diario \\
\hline 13 & Empatía 1 & Oportunidad de los comentarios \\
\hline 14 & Empatía 2 & Contribución al buen clima de trabajo \\
\hline 15 & Empatía 3 & $\begin{array}{l}\text { Conocimiento de las necesidades de tus } \\
\text { compañeros }\end{array}$ \\
\hline 16 & Empatía 4 & $\begin{array}{l}\text { Apoyo a los compañeros cuando se percibe que } \\
\text { lo necesitan }\end{array}$ \\
\hline 17 & $\begin{array}{l}\text { Relaciones } \\
\text { interpersonales } 1\end{array}$ & $\begin{array}{l}\text { Escucha atenta de la opinión de los demás para } \\
\text { lograr acuerdos }\end{array}$ \\
\hline 18 & $\begin{array}{c}\text { Relaciones } \\
\text { interpersonales } 2\end{array}$ & $\begin{array}{l}\text { Contribución a la solución de tensiones o } \\
\text { conflictos en el trabajo }\end{array}$ \\
\hline 19 & $\begin{array}{c}\text { Relaciones } \\
\text { interpersonales } 3\end{array}$ & $\begin{array}{l}\text { Interpretación de las necesidades del grupo } \\
\text { para compartirlas }\end{array}$ \\
\hline 20 & $\begin{array}{c}\text { Relaciones } \\
\text { interpersonales } 4\end{array}$ & $\begin{array}{l}\text { Intercambio de estrategias de trabajo con los } \\
\text { compañeros. }\end{array}$ \\
\hline
\end{tabular}

En este punto estudiamos la relación de las veinte variables de esta primera dimensión con la variable criterio a través del algoritmo CART. En la figura 1 se observa que los nodos más extremos son el 3 y el 12. El nodo 3 se corresponde con un grupo de 62 sujetos que se puntúan como medianamente competentes para el trabajo (media de 3,5 en el ítem 61) y se caracteriza por el hecho de que son estudiantes que se puntúan con 3 o menos en la variable 17 (relaciones interpersonales 1) y con 4 o menos en la variable 9 (motivación 1). En consecuencia, según este resultado, los sujetos que se consideran como menos competentes profesionalmente son los tienen menos confianza en que los objetivos que se plantean sean alcanzables y merezca la pena pelear por conseguirlos y, al mismo tiempo, consideran que escuchan con menos atención la opinión de los demás para lograr acuerdos. Por otra parte, el nodo 12 es el que se puntúa con una media más alta en competencia general para el trabajo (media en el ítem 61 de 5,85 sobre 6 ) y está constituido por 60 sujetos que se puntúan con más de 5 en la variable 8 (autocontrol 4), más de 5 en el ítem 12 (motivación 4) y más de 4 en el ítem 9 (motivación 1), esto es, se caracterizan por una alta motivación e interés por el trabajo y por el hecho de que consideran que sus acciones contribuyen a superar las situaciones complejas que se puedan plantear en el mismo. 


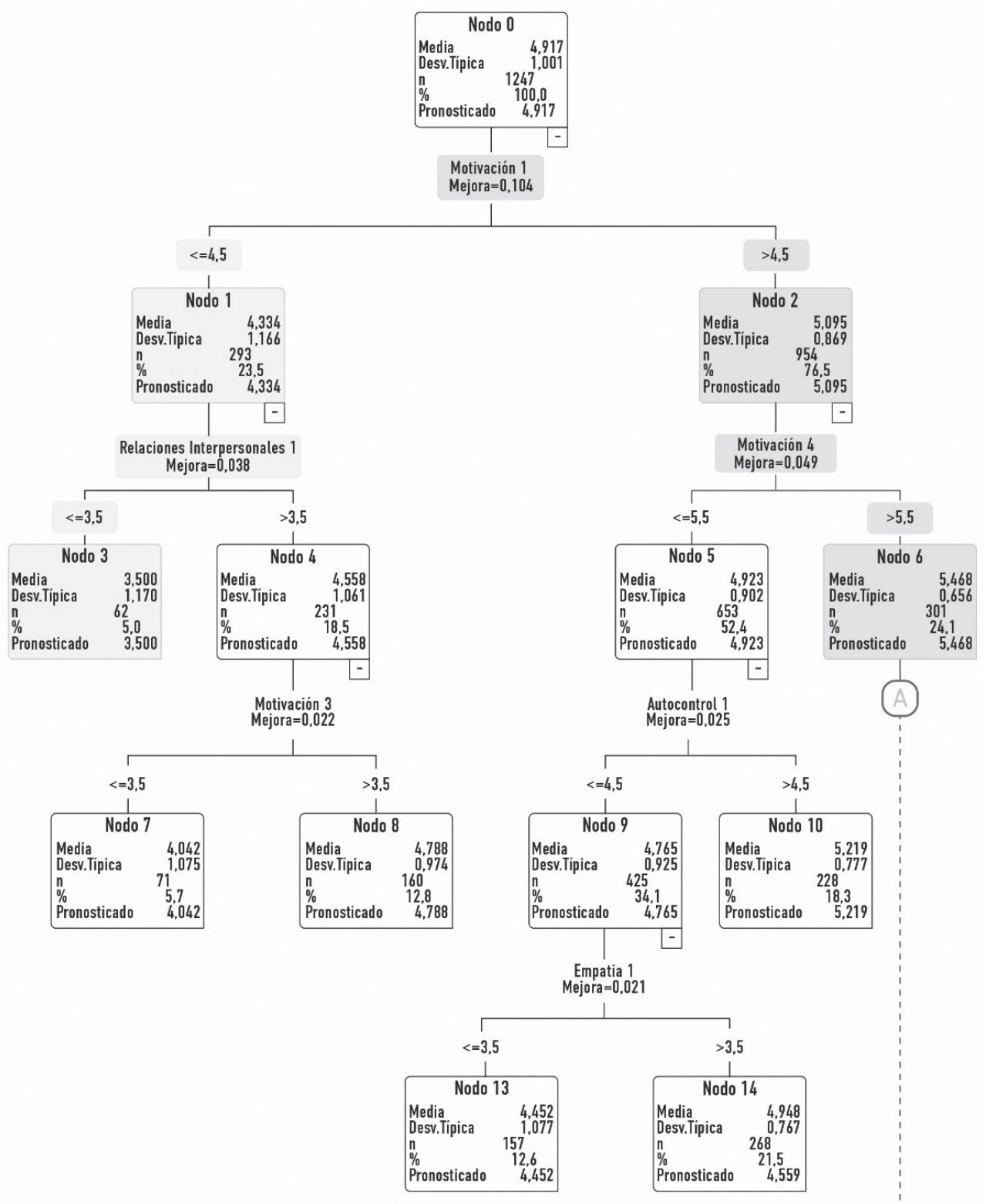




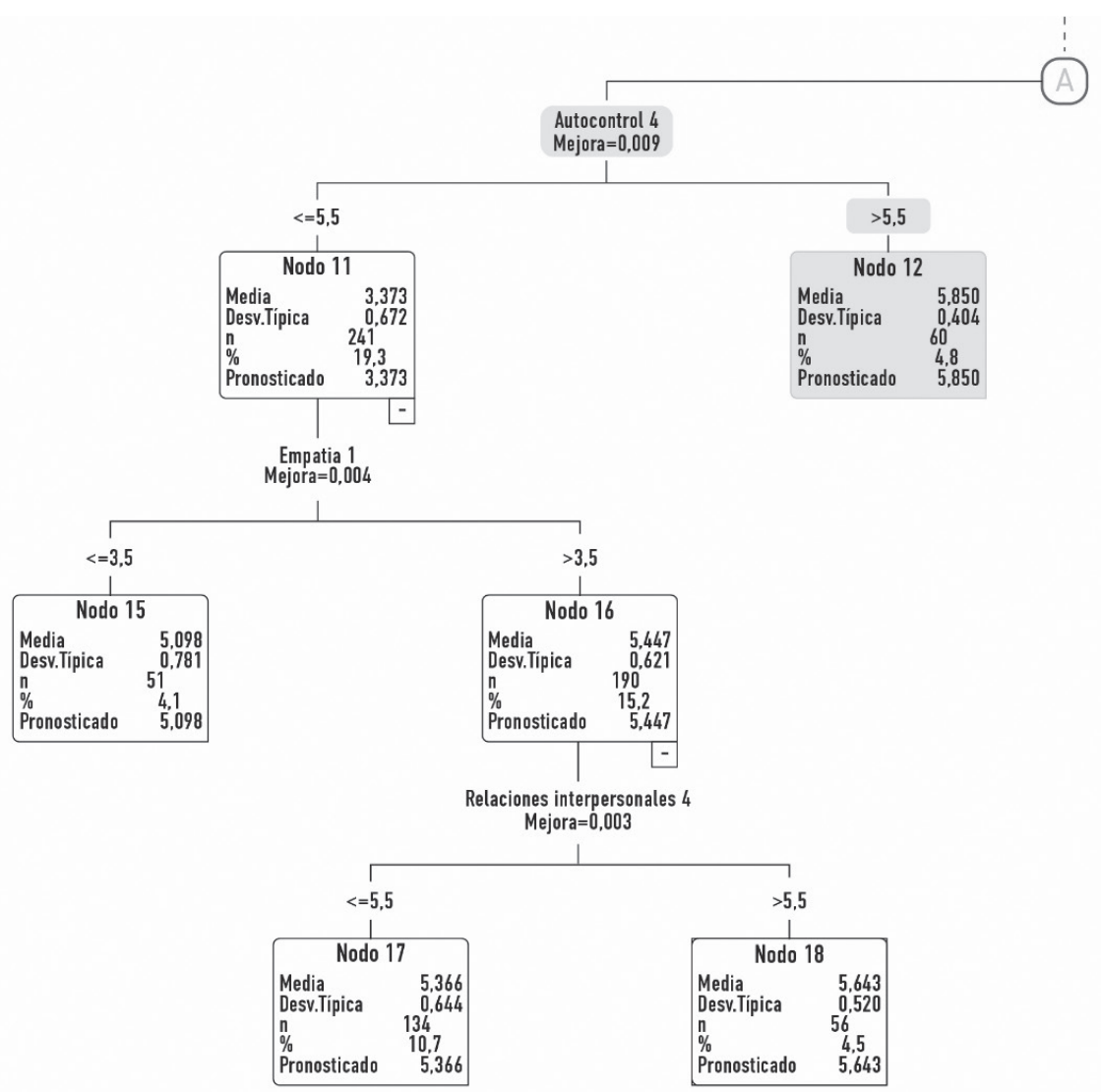

Figura 1. Árbol de regresión de competencias emocionales que más discriminan entre quienes se consideran más capaces y menos para el ejercicio profesional 


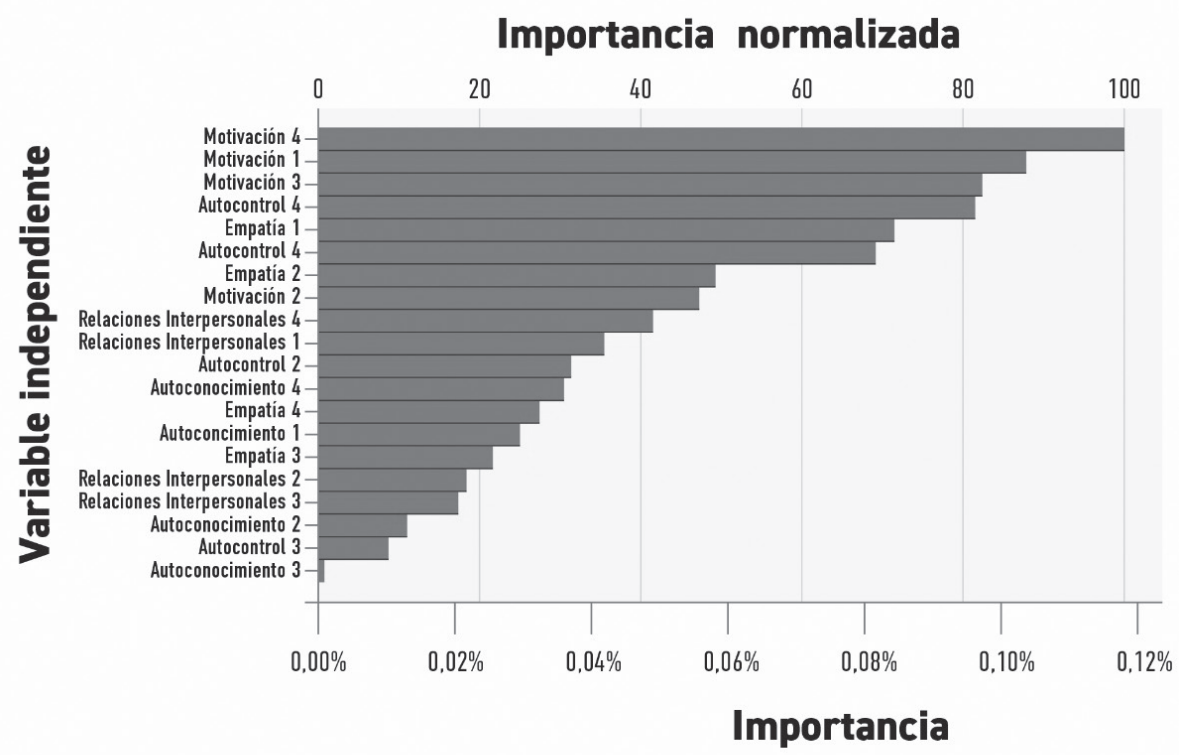

Método de crecimiento: CRT Variable dependiente: refer

Figura 2. Gráfico de importancia de las competencias emocionales para el ejercicio profesional

En la figura 2 vemos que, a la hora de pronosticar el nivel de competencia general en el trabajo a partir de las competencias emocionales, las más importantes son las relacionadas con la motivación y las menos importantes las referidas al autoconocimiento. La principal es el interés por la labor que se realiza diariamente y la más fútil para los universitarios consultados el reconocer los propios estados de ánimo.

La segunda dimensión está compuesta por las competencias de gestión, que constituyen una sub-escala con una fiabilidad de 0,919, se describen en la tabla 3.

Tabla 3

Competencias directivas

\begin{tabular}{cll}
\hline Ítem & Sub-dimensión & Descripción del ítem \\
\hline 21 & Pensamiento estratégico 1 & Visualización de escenarios futuros para la empresa \\
22 & Pensamiento estratégico 2 & $\begin{array}{l}\text { Anticipación para afrontar exitosamente los retos } \\
\text { futuros }\end{array}$ \\
23 & Pensamiento estratégico 3 & Detección de oportunidades para la empresa
\end{tabular}


24 Pensamiento estratégico 4

25 Liderazgo 1

26 Liderazgo 2

27 Liderazgo 3

28 Liderazgo 4

29 Trabajo en equipo 1

30 Trabajo en equipo 2

31 Trabajo en equipo 3

32 Trabajo en equipo 4

33 Orientación a resultados 1

34 Orientación a resultados 2

35 Orientación a resultados 3

36 Orientación a resultados 4

37 Comunicación 1

38 Comunicación 2

39 Comunicación 3

$40 \quad$ Comunicación 4
Habilidad para transformar las debilidades en fortalezas

Influencia en compañeros para el logro de las metas Fomento en compañeros del logro en momentos de crisis

Sentir como propios los objetivos de la organización Compromiso con el trabajo bien hecho Integración con compañeros para trabajar colectivamente

Colaboración para impulsar ideas innovadoras

Participación para complementar el trabajo en equipo

Trabajar buscando el equilibrio del equipo

Acierto en decisiones en momento de crisis

Reorientación del trabajo hacia el logro de las metas

Aporte de soluciones estratégicas en el trabajo diario

Buscar eficiencia de recursos para alcanzar las metas

Expresión clara de las ideas

Capacidad de convencer a los demás

Comprensión del lenguaje no verbal de los compañeros

Promoción de mensajes que favorecen un clima sano 


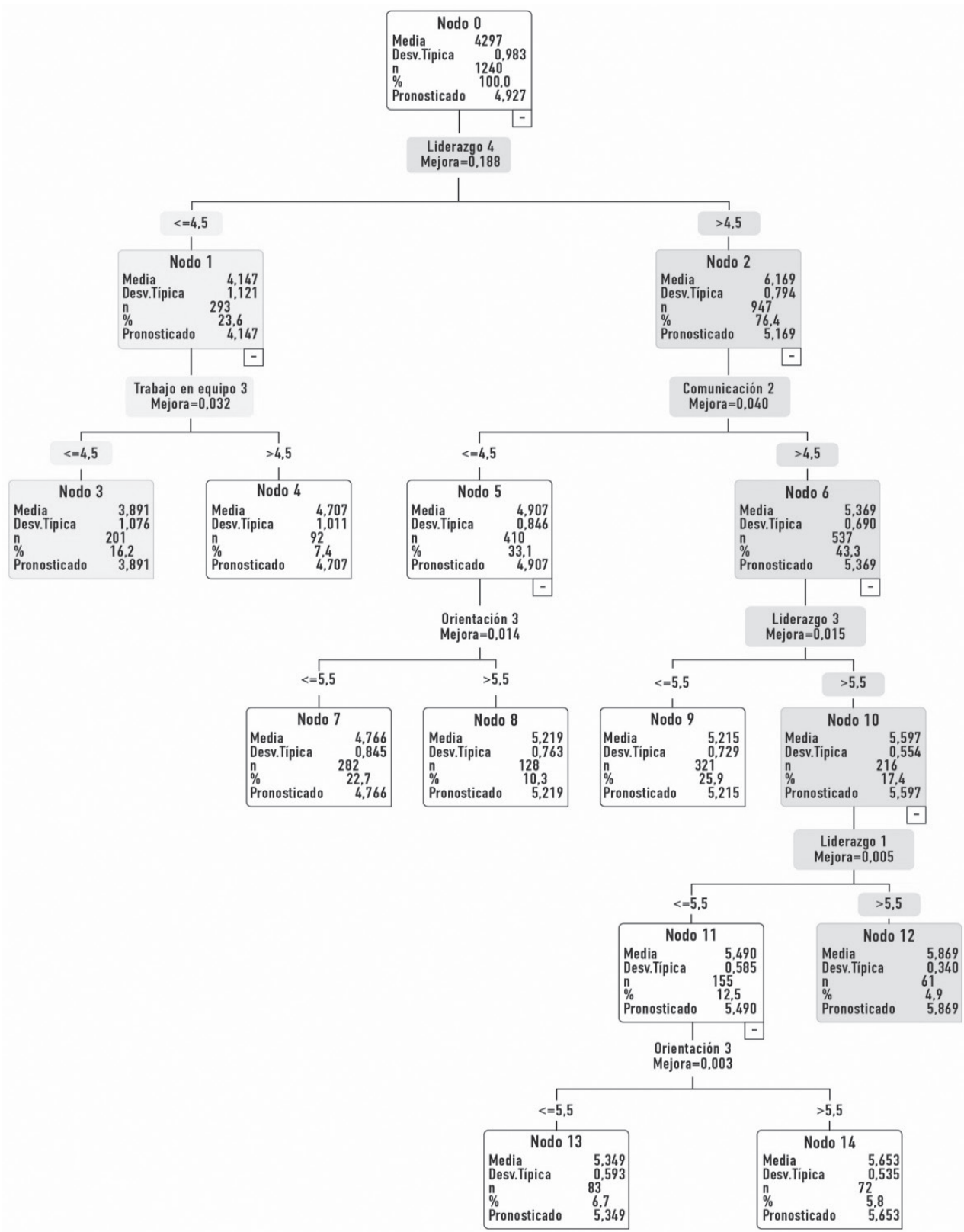

Figura 3. Árbol de regresión de competencias directivas que más discriminan entre quienes se consideran más capaces y menos para el ejercicio profesional

Cuando nos centramos en la segunda dimensión, los nodos más extremos son también el 3 y el 12. Los 201 sujetos del nodo 3 tienen una media de 3,89 en el ítem 61 o ítem criterio y se caracterizan por puntuarse a 
sí mismos con 4 o menos en la variable 3, de participación, y en la variable 28, de compromiso con la tarea. Los 61 sujetos que conforman el nodo 12 se otorgan una media de 5,87 en el criterio y se caracterizan por darse un 6 en el ítem 25 y en el ítem 27, así como por otorgarse un 5 o más en el ítem 38 y en el ítem 28. Siguiendo el perfil resultante del árbol CART obtenido, las características directivas de una alta puntuación en competencia profesional son el liderazgo de empuje y el sentimiento de los objetivos de la organización como propios, el compromiso con el trabajo bien hecho y la capacidad de convencer a los compañeros.

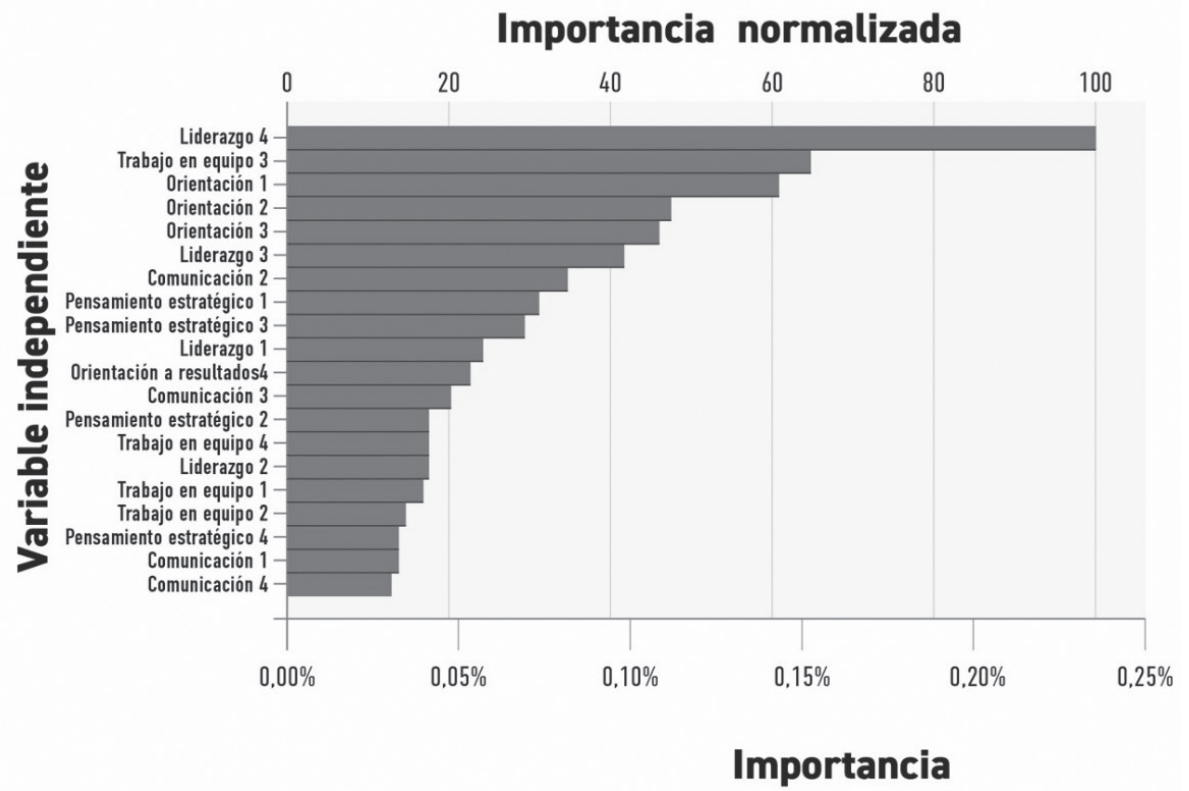

Método de crecimiento: CRT Variable dependiente: refer

Figura 4. Gráfico de importancia de las competencias directivas para el ejercicio profesional

Según la información sobre importancia normalizada aportada por CART, la variable más importante a la hora de pronosticar la variable criterio es el compromiso, seguida por la participación activa en los trabajos de equipo y el acierto en la toma de decisiones en momentos de crisis. Las menos importantes resultaron ser las variables 40,37 , la 24 , habilidad para convertir las habilidades en fortalezas, y el trabajo en equipo que impulsa innovaciones (variable 30) y el trabajo en equipo que busca la integración (variable 29).

El resto de competencias laborales, que constituyen la tercera dimensión $(a=0,876)$, se describen en la tabla 4 
Tabla 4

Competencias laborales

\begin{tabular}{|c|c|c|}
\hline Ítem & Sub-dimensión & Descripción del ítem \\
\hline 41 & Disciplina 1 & Compromiso con el trabajo diario \\
\hline 42 & Disciplina 2 & Puntual cumplimiento con los acuerdos \\
\hline 43 & Disciplina 3 & Fomento del respeto a las normas \\
\hline 44 & Disciplina 4 & Búsqueda del éxito en metas altas \\
\hline 45 & Ética 1 & Actuación ética \\
\hline 46 & Ética 2 & Promoción del respeto \\
\hline 47 & Ética 3 & Trato igualitario a los compañeros \\
\hline 48 & Ética 4 & Actuar coherente los valores \\
\hline 49 & Espíritu emprendedor 1 & Búsqueda de acciones innovadoras \\
\hline 50 & Espíritu emprendedor 2 & Impulso de nuevas formas de trabajo \\
\hline 51 & Espíritu emprendedor 3 & $\begin{array}{l}\text { Programación y seguimiento de las } \\
\text { iniciativas }\end{array}$ \\
\hline 52 & Espíritu emprendedor 4 & $\begin{array}{l}\text { Previsión de posibles resultados de la } \\
\text { acción }\end{array}$ \\
\hline 53 & $\begin{array}{l}\text { Aprendizaje y mejora } \\
\text { continua } 1\end{array}$ & Evaluación continua \\
\hline 54 & $\begin{array}{l}\text { Aprendizaje y mejora } \\
\text { continua } 2\end{array}$ & Actitud positiva ante la evaluación \\
\hline 55 & $\begin{array}{l}\text { Aprendizaje y mejora } \\
\text { continua } 3\end{array}$ & Buscar la cultura de la evaluación \\
\hline 56 & $\begin{array}{l}\text { Aprendizaje y mejora } \\
\text { continua } 4\end{array}$ & $\begin{array}{l}\text { Inversión de tiempo en la superación } \\
\text { personal }\end{array}$ \\
\hline 57 & Empeño 1 & Resiliencia en el trabajo \\
\hline 58 & Empeño 2 & Pronta superación de los fracasos \\
\hline 59 & Empeño 3 & Actuación con constancia y dedicación \\
\hline 60 & Empeño 4 & Fuerza constante para lograr un objetivo \\
\hline
\end{tabular}

En lo que se refiere a las 20 variables que constituyen la dimensión de las competencias laborales cuando se hace un árbol de clasificación tomando como variable dependiente la variable criterio (ítem 61), el logaritmo CART indica que los nodos más extremos son los nodos 3 y 14. El nodo 3 , con $n=$ 136 y una media de 3.721 en el ítem criterio, está formado por los sujetos cuyos valores otorgados fueron igual o menores a 3 en el ítem 44 y de 4 o menos en el ítem 59. De acuerdo a este resultado son los participantes que se han valorado como menos exitosos al cumplir tareas con objetivos altos, 
así como los que se han valorado con menor habilidad en la persistencia hacia el logro de las tareas.

El nodo óptimo es el 14, con una media de casi 5,45 en el ítem criterio y constituido por $n=152$ participantes, que se puntúan con valores de 5 y mayor en el ítem 49, de innovación, se otorgan valores de 3 o más en el ítem 54, esto es, tienen actitud positiva hacia la evaluación, se dan valores menores de 6 para el ítem 44, de compromiso personal, de 3 o menos en el ítem 60 y de más de 5 en el ítem 59, que mide perseverancia en el trabajo. Las variables de esta dimensión más importantes, a la hora de explicar la variable criterio de competencia global percibida, son el ítem 59, seguida del ítem 44 y las variables menos importantes son el ítem 47 y el ítem 55, en los que los participantes se autovaloran en imparcialidad y realización de acciones de mejora continua. 


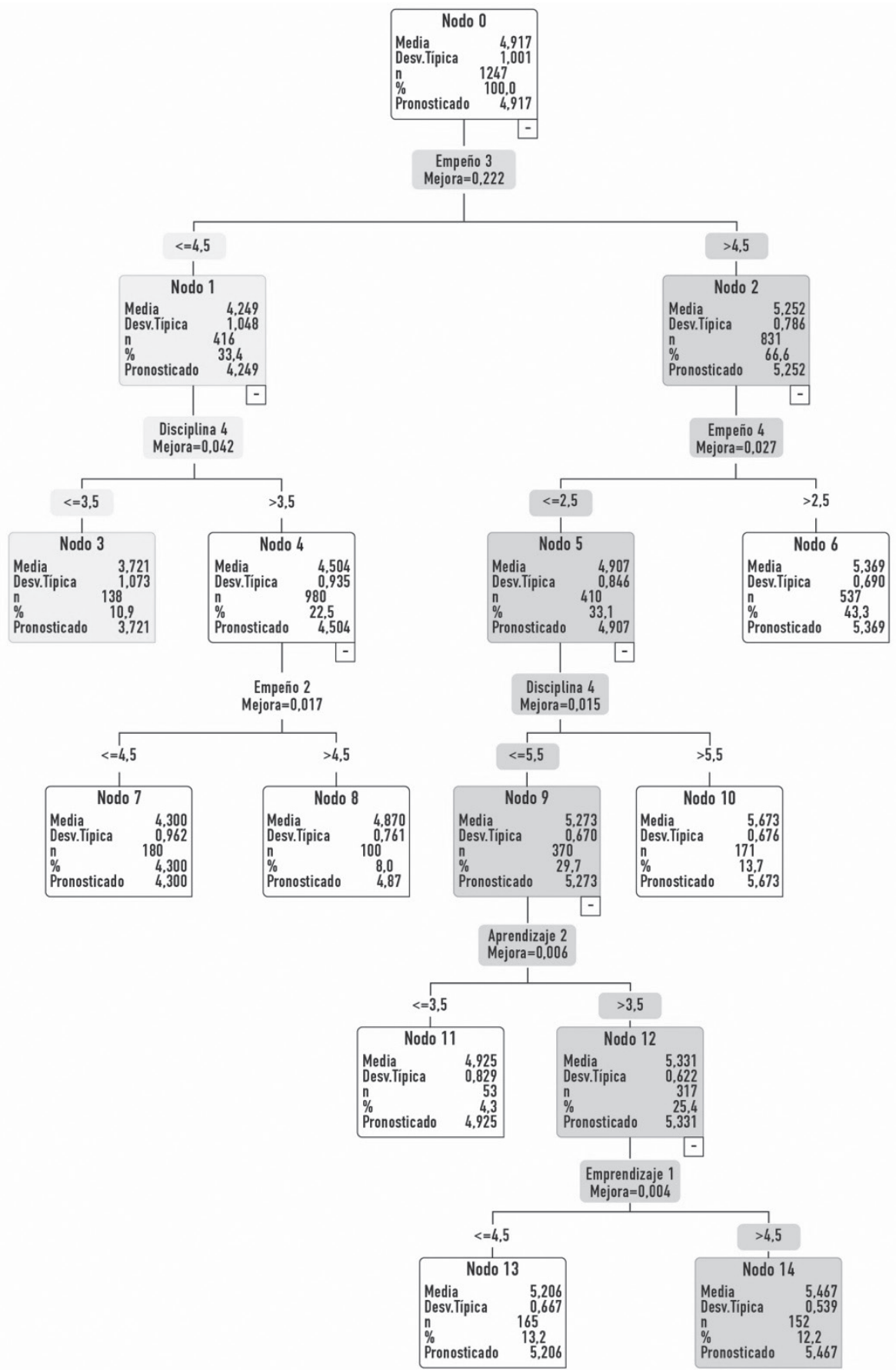

Figura 5. Árbol de regresión de competencias laborales que más discriminan entre quienes se consideran más capaces y menos para el ejercicio profesional 


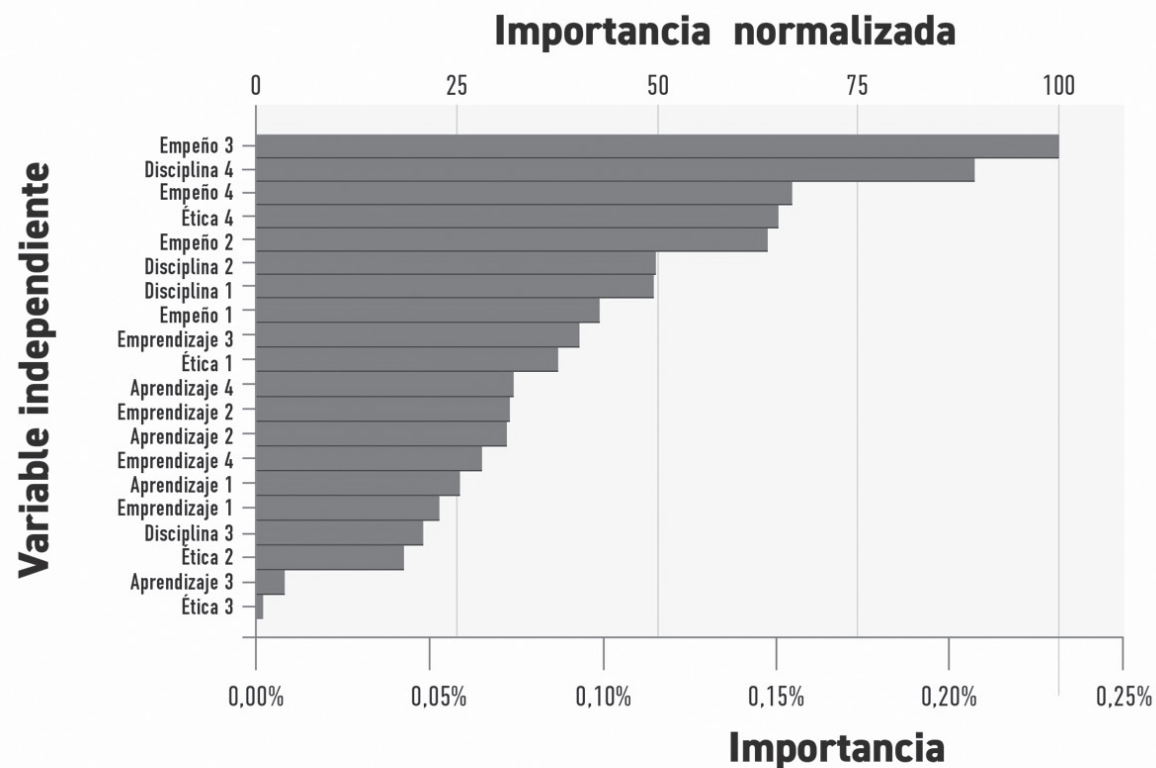

Método de crecimiento: CRT Variable dependiente: refer

Figura 6. Gráfico de importancia de las competencias laborales del ejercicio profesional

\section{Competencias explicativas del nivel global competencial auto- percibido}

Con el propósito de ubicar, de las 60 que componen el cuestionario, las principales variables en cuanto a su capacidad de explicación del ítem elegido como criterio, se ha obtenido, del análisis de segmentación mediante el algoritmo CART, que los nodos más extremos son el 3 y el 8 . El nodo 3 se corresponde con un grupo con $\mathrm{N}=188$ sujetos que se puntúan como medianamente competentes para el trabajo, con una media de 3.72 y se caracteriza por el hecho de que son sujetos que se puntúan como menos de 4.5 en el ítem 59 y en el ítem 28 , que emergen como las variables de más importancia a la hora de explicar la variable dependiente o variable criterio. De esta manera, los participantes que se perciben como menos competentes globalmente son los que se consideran menos persistentes hacia el logro de las tareas y los que confiesan que se comprometen menos con realizar bien el trabajo, siendo la puntuación de corte en ambos casos 4,5.

El nodo 8 está formado por 160 participantes que se puntúan con 6 (siempre se realiza) en el ítem 44 y en el ítem 28 y con 5 o 6 en el ítem 59 . Se caracterizan por tener altura de metas, comprometerse con el trabajo bien hecho y ser persistentes en el logro de los objetivos que se han propuesto. 
En consecuencia, las variables que más peso tienen en la explicación de la competencia global autopercibida (ítem 61) son el ítem 28, 59 y 44. La menos explicativa es el ítem 55, variable de participación en cursos de capacitación o entrenamiento para el aprendizaje y mejora continua.

En la tabla 5 se ofrecen ordenadas 20 de las 60 variables que componen el COMPETEST según su importancia en la explicación de la competencia para el trabajo globalmente autopercibida, tomada como variable criterio o referencia y medida con la incorporación en el cuestionario del ítem 61. Entre las 10 variables más relacionadas con la variable criterio se encuentran 6 laborales (tres ítems de empeño, dos de disciplina y uno de ética), 3 directivas (uno de orientación a la meta, uno de liderazgo, uno trabajo en equipo y uno comunicación interpersonal) y 1 emocional (un ítem de motivación).

Entre las 10 variables menos importantes para la variable de referencia hay 6 de la dimensión de competencias emocionales (dos de autoconocimiento, uno de relaciones interpersonales, dos de empatía y uno de autocontrol), 2 competencias laborales (un ítem de la sub-dimensión de ética y otro de la de aprendizaje y mejora continua) y 2 competencias directivas (un ítem de pensamiento estratégico referido a la habilidad para transformar las debilidades en fortalezas y uno de comunicación interpersonal). 


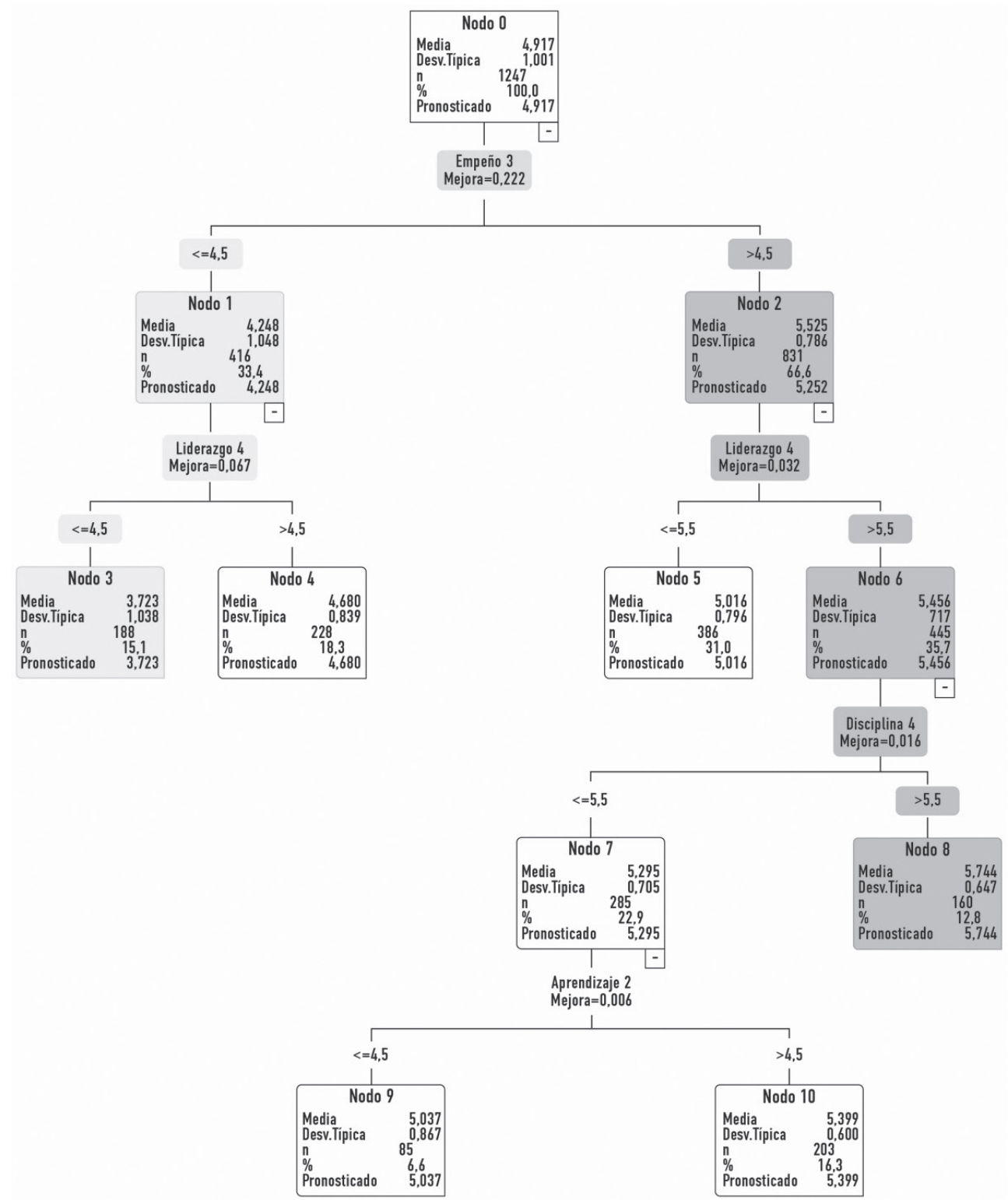

Figura 7. Árbol de regresión del nivel global competencial 
Tabla 5

Competencias genéricas más importantes y más fútiles

\begin{tabular}{|c|c|c|c|}
\hline \multicolumn{2}{|c|}{ Variable independiente } & \multirow[t]{2}{*}{ Importancia } & \multirow[t]{2}{*}{$\begin{array}{l}\text { Importancia } \\
\text { normalizada }\end{array}$} \\
\hline Item & Dimensión & & \\
\hline 28 & C. DIRECTIVA & 0,286 & $100,00 \%$ \\
\hline 59 & C. LABORAL & 0,248 & $86,60 \%$ \\
\hline 44 & C. LABORAL & 0,212 & $73,90 \%$ \\
\hline 58 & C. LABORAL & 0,167 & $58,20 \%$ \\
\hline 41 & C. LABORAL & 0,165 & $57,70 \%$ \\
\hline 48 & C. LABORAL & 0,163 & $56,90 \%$ \\
\hline 60 & C. LABORAL & 0,163 & $56,80 \%$ \\
\hline 9 & C. EMOCIONAL & 0,158 & $55,00 \%$ \\
\hline 31 & C. DIRECTIVA & 0,153 & $53,60 \%$ \\
\hline 36 & C. DIRECTIVA & 0,149 & $52,00 \%$ \\
\hline 16 & C. EMOCIONAL & 0,036 & $12,70 \%$ \\
\hline 4 & C. EMOCIONAL & 0,034 & $11,70 \%$ \\
\hline 47 & C. LABORAL & 0,029 & $10,30 \%$ \\
\hline 19 & C. EMOCIONAL & 0,028 & $9,70 \%$ \\
\hline 24 & C. DIRECTIVA & 0,027 & $9,50 \%$ \\
\hline 40 & C. DIRECTIVA & 0,020 & $6,90 \%$ \\
\hline 15 & C. EMOCIONAL & 0,018 & $6,20 \%$ \\
\hline 7 & C. EMOCIONAL & 0,017 & $6,10 \%$ \\
\hline 2 & C. EMOCIONAL & 0,017 & $5,90 \%$ \\
\hline 55 & C. LABORAL & 0,012 & $4,10 \%$ \\
\hline
\end{tabular}

\section{DISCUSIÓN Y CONCLUSIONES}

El análisis de segmentación mediante el algoritmo CART ha aportado algunas evidencias acerca de la capacidad de las medidas obtenidas con el COMPETEST para predecir la competencia profesional global a partir de las competencias genéricas incluidas en el cuestionario y para discriminar entre quiénes se perciben como más y menos competentes para el ejercicio profesional. De acuerdo con Hernández, Ramírez, y Ferri (2007) los árboles de decisión ponen de manifiesto una tendencia, marcada por la regularidad existente en los datos, que facilita la toma de decisiones más apropiada de 
acuerdo a dicha tendencia. Es cierto que se puede considerar como una limitación el que no aporten un índice numérico que permita cuantificar el grado de validez predictiva de la medida, pero sin duda, arrojan una información pertinente y útil de cara a la elección de predictores y su importancia relativa. Digamos que dibujan un modelo predictivo de alto valor informativo a partir del cual se pueden hacer estudios en profundidad.

En el ámbito en el que se ha realizado esta investigación con los árboles de decisión se han identificado las competencias genéricas más relacionadas con la competencia profesional autopercibida. Entre ellas, algunas competencias emocionales han resultado relevantes, aunque, cuando se incluyen en el modelo junto con otro tipo de competencias profesionales y de gestión, la que se mantiene en los puestos más relevantes es la claridad de objetivos. Los autores concuerdan en la necesidad del desarrollo de la inteligencia emocional (Goleman, 2010; Saarni, 2000; Bar- On, 2006) y desde la psicología el debate se centra en la definición y dimensionalidad del constructo. Desde la pedagogía, aunque la base científica del constructo nos atañe, nos interesa más la perspectiva aplicada que se traduce en la identificación de las competencias relevantes para los fines que se persiguen en cada nivel educativo y el desarrollo de las estrategias y procesos para su dominio y en este estudio ha emergido, como una competencia emocional valorada como muy importante por los participantes, la dimensión de motivación. Por otra parte, de cara a potenciar la empleabilidad de los estudiantes, emergen también algunas competencias emocionales que, habiéndose incluido en el cuestionario porque los empleadores las consideraban importantes, para los encuestados no resultan especialmente relacionadas con su autovaloración profesional global. El estudio empírico realizado sitúa como menos importantes para el empleo algunas competencias emocionales relacionadas con el autoconocimiento, el autocontrol, la empatía o las relaciones interpersonales. De las competencias directivas más valoradas cabe destacar la búsqueda de eficiencia y el espíritu de trabajo en equipo. Pero, consistentemente con el criterio empírico utilizado, son las competencias laborales las que emergen como más importantes. Son las incluidas en la tercera dimensión del cuestionario, que se refieren de manera más directa a lo laboral. De ellas, las de empeño, disciplina y ética aparecen como las más relevantes, siendo aspectos que habría que plantearse trabajar en la Universidad de manera intencional y estratégica si se busca mejorar la empleabilidad de los estudiantes.

Queda como prospectiva de investigación estudiar cómo actuar para el logro en la Universidad del dominio de las competencias genéricas más relevantes. López, León y Pérez (2018) concluyen que el profesorado se encuentra con dificultades para incorporar este tipo de competencias en 
el currículum académico y considera que el éxito de un modelo formativo basado en competencias puede depender de la adopción de perspectivas más holísticas. La consideración del deporte universitario como una actividad formativa trasversal que apoye el trabajo realizado en el aula puede ser a nuestro juicio una alternativa viable y eficaz en esta línea. Hay que estudiar de manera detallada la relación de la práctica deportiva con las diferentes competencias identificadas para establecer cuáles de ellas podrían potenciarse de manera estratégica a partir de una planificación de la formación en competencias basada en la evidencia. Pero existen algunos trabajos que nos permiten plantear hipótesis acerca de los efectos beneficiosos que una práctica deportiva adecuadamente organizada podría tener. Hold y Dunn (2004) obtienen que el deporte desarrolla habilidades como la disciplina, el espíritu de sacrificio o el compromiso, definido como la fuerza de la motivación y el carácter para cumplir con los objetivos fijados. Ruiz y Cabrera (2004) identifican como un valor que un deportista posee, el autocontrol mostrado en acciones positivas que ayudan a superar un momento de tensión en el trabajo, que es otro rasgo que los indicadores estadísticos han marcado como relevante. Gould, Dieffenbach y Moffett, (2002) destacan como características que tienen que ver con la resistencia mental de los deportistas, la capacidad para recuperarse de los fracasos o para afrontar la presión. Otros factores relacionados con el deporte son el logro del equilibrio necesario entre los miembros del equipo, permaneciendo alerta ante las acciones que se van a emprender y cumpliendo puntualmente con lo establecido pese a los obstáculos que pudieran encontrarse (Weinberg \& Gould, 2010; Bull, Shambrook, James, \& Brooks,2005; Thelwell, Westony, \& Greenlees, 2005).

Este artículo es solo una parte de una investigación más amplia sobre la relación del deporte y las competencias genéricas y en este sentido no aborda cuestiones esenciales que se presentarán y discutirán en otros trabajos. Pero aun así representa una aportación en la medida en que puede contribuir a llegar a entender qué competencias resultan claves en la empleabilidad y en la formación universitaria necesaria para mejorar este indicador de calidad de las instituciones de educación superior.

La utilización de los árboles de regresión en investigación educativa y en el estudio particular de la formación en competencias representa una aproximación metodológica novedosa y prometedora, puesto que viene a complementar otros enfoques de tratamiento de datos menos holísticos y al tiempo más exigentes en cuanto al nivel de medida de las variables que se analizan. El estudio de la validez predictiva de las medidas que más usualmente empleamos en educación se enriquece a nuestro juicio con el empleo de esta técnica multivariada, que viene a aportar evidencias de validez basadas en un enfoque integrador de los clásicos índices de validez de 
los ítems, que de manera analítica se conforman con calcular la correlación de cada ítem con la variable criterio.

\section{NOTA}

Este trabajo se realiza con el apoyo de beca CONACYT-México para la tesis doctoral (Álvarez-Benítez, 2016) de la que este artículo es una publicación. 


\section{REFERENCIAS BIBLIOGRÁFICAS}

\begin{abstract}
American Educational Research Association (AERA), American Psychological Association (APA) and National Council on Measurement in Education (NCME) (2014). Standards for Educational and Psychological Testing. APA.
\end{abstract}

Álvarez-Benítez, M. (2016). La práctica deportiva en las instituciones de Educación Superior como herramienta para la formación en competencias genéricas. [Tesis doctoral]. UCM

Álvarez-Benítez, M., Asensio-Muñoz, I., y García-Ramos, J.M. (2013). Deporte y competencias genéricas en la Universidad. Revista Complutense de Educación, 24(1), 141-163.

Artinger, L., Clapham, L., Hunt, C., Meigs, M., Milord, N., Sampson, B., \& Forrester, S.A. (2006). The Social Benefits of Intramural Sports. Journal NASPA-Student Affairs Administrators in Higher Education, 43 (1), 69-86.

Asensio, I., Carpintero, E., López, E., y Expósito, E. (2018). ¿Cuánto oro hay entre la arena? Minería de datos con los resultados de España en PISA 2015, Revista Española de Pedagogía, 270, 225-245. https://doi.org/10.22550/ REP76-2-2018-02

Bar-On, R. (2006). The Bar-On model of emotional-social intelligence (ESI). Psicothema, 18, 13-25

Berlanga, V., Rubio, M. J., y Vilá, R. (2013). Cómo aplicar árboles de decisión en SPSS. REIRE, Revista d'Innovació $i$ Recerca en Educació, 6(1), 65-79

Blanco, A., Asensio, I., Carpintero, E., Ruiz de, C., \& Expósito, E. (2017). Aplicaciones de la Segmentación Jerárquica en Medición y Evaluación de Programas Educativos. Ejemplos con un Programa De Educación Financiera. Educación XX1, 20(2), 235-257. https://doi.org/10.5944/ educxx1.19039

Borda, J., y Ormeño, J.M. (2010). Rendimiento deportivo de la Universidad Nacional Mayor de San Marcos en los juegos deportivos universitarios nacionales de Trujillo 2006 y Tacna 2008. Revista Investigación Educativa, 14 (25), 155181.

Breiman, L., Friedman, J.H., Olshen R.A., \& Stone, C.J. (1984). Classification and regression trees. Chapman \& Hall/CRC.

Bull, S. Shambrook, C., James, W., \& Brooks, J. (2005). Toward an understanding of mental toughness in elite. English cricketers. Journal of applied Sport Psychology, 17, 209-227.

Castro, M. (2011). ¿Qué sabemos de la medida de las competencias? Características y problemas psicométricos en la evaluación de competencias. Bordón. Revista de Pedagogía, 63(1), 109-123.

Castro, M., y Lizasoaín, L. (2012). Las técnicas de modelización estadística en la investigación educativa: minería de datos, modelos de ecuaciones estructurales y modelos jerárquicos lineales. Revista Española de Pedagogía, 70(251), 131-148.

Goleman, D. (2010) Inteligencia Emocional. ( $77^{\circ}$ Edición). Kairos

Goleman, D. (2007). La Práctica de la Inteligencia Emocional (20 a . Edición). Kairos.

González, V. y González, R (2008). Competencias genéricas y formación profesional: un análisis desde la 
docencia universitaria. Revista Iberoamericana de Educación, 47, 185209.

Gould, D., Dieffenbach, K., \& Moffett, A. (2002). Psychological Characteristics and Their Development in Olympic Champions. Journal of Applied Sport Psychology. 14(3), 172-204.

Hair, J.F., Anderson, R.E., Tatham, R.L. y Black, W. (1999). Análisis multivariante. (5 ${ }^{\mathrm{a}}$ Edición). Prentice Hall

Hernández, J., Ramirez, J., y Ferri, C. (2007). Introducción a la Minería de Datos. Pearson.

Holt, N.L., \& Dunn, J. G.H. (2004). Toward a Grounded Theory of the Psychosocial Competencies and Environmental Conditions Associated with Soccer Success. Journal of Applied Sport Psychology, 16(3), 199-219.

Lizasoain, L., y Joaristi, L. (2000). El análisis de datos en la evaluación de programas educativos. Revista de Investigación Educativa, 18(2), 357379.

Lizasoain, L., Joaristi, L., Santiago, C, Lukas, J.F., Moyano N., Sedano, M., y Munárriz, B. (2003). El uso de las técnicas de segmentación en la evaluación del rendimiento en lenguas. Un estudio en la Comunidad Autónoma Vasca. Revista de Investigación Educativa, 21(1), 93-111.

López López, M.C.; León Guerrero, M.J., y Pérez García, P. (2018). El enfoque por competencias en el contexto universitario español. La visión del profesorado. Revista de Investigación Educativa, 36(2), 529-545. http:// dx.doi.org/10.6018/rie.36.2.314351

Martínez Abad, F., \& Chaparro Caso López, A.A. (2017). Data-mining techniques in detecting factors linked to academic achievement, School Effectiveness and School Improvement, 28(1), 39-55. https://doi.org/10.1080/09 243453.2016.1235591

Morales, P., Urosa, B., y Blanco, A. (2003). Construcción de escalas de actitudes tipo Likert. La Muralla

Romero, C., \& Ventura, S. (2010). Educational data mining: A review of the state of the art. IEEE Transactions on Systems, Man, and Cybernetics-Part C: Applications and Reviews, 40(6), 601-618.

Ruiz Llamas, G., y Cabrera Suárez, D. (2004). Los valores en el deporte. Revista de Educación, 335, 9-19.

Saarni, C. (2000). Emotional Competence; A Developmental Perspective. En R. Bar On \& J. D.A. Parker (Eds.). The Handbook of Emotional Intelligence. (pp. 68-91), Jossey - Bass.

Sireci, S., \& Padilla, J.L. (2014). Validiting assessment: Introduction to the Special Section. Psicothema, 26, 97-99

Thelwell, R., Weston, N., \& Greenlees, I. (2005). Defining and understanding mental toughness within soccer. Journal of Applied Sport Psychology, $17,326-332$

Tourón, J., López-González, E., Lizasoain, L., Navarro, E., y García, M.J. (2018). Alumnado español de alto y bajo rendimiento en ciencias en PISA 2015: análisis del impacto de algunas variables de contexto. Revista de Educación, 380, 156184. https://doi.org/10.4438/1988592X-RE-2017-380-376

Weinberg, R.S., \& Gould, D. (2010). Introducción al entrenamiento de las habilidades Psicológicas. En R. S. Weinberg y D. Gould, Fundamentos 
de Psicología del Deporte y del Ejercicio

Físico (4a . Edición). (pp. 249-272).

Panamericana. 


\section{PERFIL ACADÉMICO Y PROFESIONAL DE LAS AUTORAS}

\section{María Mónica Álvarez. ORCID}

Doctora en Educación por la Universidad Complutense de Madrid. Colaboradora en la UPAEP Universidad Popular Autónoma del Estado de Puebla Campus Tehuacán, México. E-mail: mariamonika.alvarez@gmail. com

Inmaculada Asensio-Muñoz. ORCID

Profesora Titular del Área de Métodos de Investigación y Diagnóstico en Educación, del Departamento de Investigación y Psicología en Educación de la UCM. Miembro del Grupo de Investigación Medida y Evaluación de Sistemas Educativos (Grupo MESE). Línea de trabajo: métodos de investigación y evaluación en educación. E-mail: macu@edu.ucm.es

Fecha Recepción del Artículo: 16. Octubre. 2019

Fecha Aceptación del Artículo: 29. Noviembre. 2019

Fecha Revisión para Publicación: 23. Enero. 2020 